\title{
Sequential Regeneration of Charmonia in Heavy-Ion Collisions
}

\author{
Xiaojian $\mathrm{Du}^{*}$ and Ralf Rapp \\ Cyclotron Institute and Department of Physics and Astronomy, \\ Texas AEM University, College Station, TX 77843-3366, USA
}

(Dated: September 16, 2015)

\begin{abstract}
We investigate the production of $\psi(2 S)$ in nuclear collisions at RHIC and LHC energies. We first address charmonium production in $200 \mathrm{GeV}$ d-Au collisions at RHIC; the strong suppression of $\psi^{\prime}$ mesons observed in these reactions indicates mechanisms beyond initial cold nuclear matter effects. We find that a more complete treatment of hadronic dissociation reactions leads to appreciable $\psi^{\prime}$ suppression in the thermal medium of an expanding fireball background for $\mathrm{d}-\mathrm{Au}$ collisions. When implementing updated hadronic reaction rates into a fireball for $2.76 \mathrm{TeV} \mathrm{Pb}-\mathrm{Pb}$ collisions at $\mathrm{LHC}$, the regeneration of $\psi^{\prime}$ mesons occurs significantly later than for $J / \psi$ 's. Despite a smaller total number of regenerated $\psi^{\prime}$, the stronger radial flow at their time of production induces a marked enhancement of their $R_{\mathrm{AA}}$ relative to $J / \psi$ 's in a transverse-momentum range of $p_{t} \simeq 3-6 \mathrm{GeV}$. We explore the consequences and uncertainties of this "sequential regeneration" mechanism on the $R_{\mathrm{AA}}$ double ratio and find that it can reproduce the trends observed in recent CMS data.
\end{abstract}

Keywords: Quark-gluon plasma; Charmonia; Ultrarelativistic heavy-ion collisions

PACS numbers: 25.75.-q, 24.85.+p, 12.38.Mh, 14.40.Lb

\section{INTRODUCTION}

Charmonium production in ultra-relativistic heavy-ion collisions (URHICs) has been studied for over 30 years. The originally proposed $J / \psi$ suppression signature of Quark-Gluon Plasma (QGP) formation [1] has evolved into a more complex problem where both suppression and so-called regeneration (or statistical hadronization) mechanisms need to be considered. Their interplay and relevance depend on collision energy, system size and the 3-momentum of the measured charmonia, see, e.g., Refs. 2-4] for recent reviews. The phenomenological modeling of these mechanisms, and their relation to the underlying in-medium properties, has progressed significantly in recent years. In particular, kinetic transport approaches, when calibrated with existing data from SPS and RHIC, have predicted the main features of the $J / \psi$ production observed in the new energy regime at the LHC [5-7] (although significant uncertainties due to, e.g., the open-charm cross section persist [8]). These include the overall increase of the nuclear modification factor, $R_{\mathrm{AA}}$, compared to RHIC energies and its enhancement at low transverse momentum, $p_{t}$. [9]

Much less is known about the $2 S$ excited state, $\psi^{\prime}(3686)$. Its small "binding" energy of about $45 \mathrm{MeV}$ (relative to the $D \bar{D}$ threshold) renders controlled theoretical calculations of its in-medium properties (binding energy and inelastic reaction rates) challenging. Experimentally, the $\psi^{\prime}$ over $J / \psi$ ratio has been measured at the SPS [10], where it was found to drop by up

* Corresponding author.

E-mail address:xjdu@physics.tamu.edu to a factor of 3 in central $17.3 \mathrm{GeV} \mathrm{Pb}-\mathrm{Pb}$ collisions. This is consistent with the statistical hadronization approach [11], but it can also be explained by transport approaches with large inelastic reaction rates of the $\psi^{\prime}$ in the hadronic phase [12, 13]. More recently, $\psi^{\prime}$ data have become available for $0.2 \mathrm{TeV}$ d-Au collisions at RHIC [14] and $5.02 \mathrm{TeV}$ p-Pb collisions at LHC [15]. $\psi^{\prime}$ mesons were found to be significantly more suppressed than $J / \psi$ mesons, which is difficult to reconcile with initial coldnuclear-matter $(\mathrm{CNM})$ effects since the passing time of the highly Lorentz-contracted incoming nuclei is much smaller than the formation time scale of the charmonia. Consequently, final-state effects have been put forward to explain these data, e.g., using the comover suppression model [16]. The latter achieves a good description of the collision energy and rapidity dependence of $\psi^{\prime}$ and $J / \psi$ production in $\mathrm{d}-\mathrm{Au}$ and $\mathrm{p}-\mathrm{Pb}$ collisions including expected shadowing effects on the parton distribution functions (see also Ref. [17]).

However, rather unexpected results have emerged from recent measurements by the CMS collaboration [18] for the double-ratio of the nuclear modification factor, $R_{\mathrm{AA}}$, of $\psi^{\prime}$ over $J / \psi$ in $2.76 \mathrm{TeV} \mathrm{Pb}-\mathrm{Pb}$ collisions at the $\mathrm{LHC}$ (preliminary results are also available from ALICE [19]). At slightly forward rapidities, $1.6<|y|<2.4$, and for transverse momenta $3<p_{t}<30 \mathrm{GeV}$, this double ratio is around $0.9 \pm 0.45 \pm 0.3$ for semi-central collisions (similar for peripheral ones), but significantly exceeds one for central collisions, $2.3 \pm 0.5 \pm 0.35$. Especially the latter has evaded any model explanations thus far, see, e.g., the detailed studies in Ref. [20]. On the other hand, around midrapidity, and for momenta $6.5<p_{t}<30 \mathrm{GeV}$, a double ratio of around $\sim 0.5$ is found, which is much more in line with common expectations of a stronger suppression of $\psi^{\prime}$ due to its much weaker binding relative to the $J / \psi$. 
In the present paper we put forward a potential mechanism to (partially) resolve the above "puzzle". Based on the rather large inelastic reaction rates for the $\psi^{\prime}$ in hadronic matter that we deduce from its suppression in $\mathrm{d}-\mathrm{Au}$ (also in line with the aforementioned SPS data), we argue that the inverse reactions of $\psi^{\prime}$ formation in $\mathrm{Pb}-\mathrm{Pb}$ collisions must also happen in the later, hadronic stages of the fireball evolution. In particular, the $\psi^{\prime}$ regeneration processes happen later than those for the $J / \psi$ whose much larger binding energy leads to an earlier "freezeout" than for the $\psi^{\prime}$. A consequence of such a "sequential freezeout" is that the collective expansion velocity of the medium leads to harder $p_{t}$ spectra for the $\psi^{\prime}$. Thus, in terms of the $R_{\mathrm{AA}}$, the $\psi^{\prime}$ can outshine the $J / \psi$ in a momentum range of $p_{t} \gtrsim M_{\psi}$, which happens to coincide with the lower CMS $p_{t}$ cut. On the other hand, at higher $p_{t}$, the regeneration contribution ceases giving way to a more sequential-like suppression pattern of primordially produced charmonia.

Our paper is organized as follow: In Sec. II we revisit our hadronic reaction rates for both $\psi^{\prime}$ and $J / \psi$, apply them within a schematic fireball for d-Au collisions at RHIC and compare our results to PHENIX data. In Sec. III we implement the updated hadronic rates in our earlier constructed transport approach. In particular, we estimate the time windows for sequential charmonium regeneration, and elaborate the uncertainty for the underlying charmonium $p_{t}$ spectra in the context of the CMS data for the $\psi^{\prime} / J / \psi R_{\mathrm{AA}}$ double ratio. We conclude in Sec. IV

\section{HADRONIC DISSOCIATION OF CHARMONIA}

Dissociation rates of charmonia in hadronic matter are usually considered to be much smaller than in the QGP (see, e.g., the discussion in Ref. [2]). However, for the $\psi^{\prime}$ this is not so obvious, since the proximity of its mass to the $D \bar{D}$ threshold provides a large phase space for break-up reactions. In the following, we revisit hadronic reactions rates for $J / \psi$ and $\psi^{\prime}$ mesons based on effective meson Lagrangians (Sec. IIA) and evaluate their consequences for final-state effects in $\mathrm{d}-\mathrm{Au}$ reactions at $\mathrm{RHIC}$ (Sec. II B).

\section{A. Update on Dissociation Rates}

Our starting point is the previously employed hadronic reaction rate 13] based on flavor- $S U(4)$ meson exchange models 21, 22] for the processes $J / \psi+\rho \rightarrow D+\bar{D}, D^{\star}+$ $\bar{D}^{\star}$ (exothermic for $m_{J / \psi}+m_{\rho}>m_{D}+m_{\bar{D}}$ and endothermic for $\left.m_{J / \psi}+m_{\rho}<m_{D^{\star}}+m_{\bar{D}^{\star}}\right)$ and $J / \psi+\pi \rightarrow$ $D^{\star}+\bar{D}, D+\bar{D}^{\star}$ (endothermic). For those reactions, the $J / \psi$ dissociation rate at $T=170 \mathrm{MeV}$ amounts to 1 $2 \mathrm{MeV}$, corresponding to a lifetime of $100-200 \mathrm{fm} / c$. Even with the typical uncertainties (factor $\sim 2-3$ ) associated
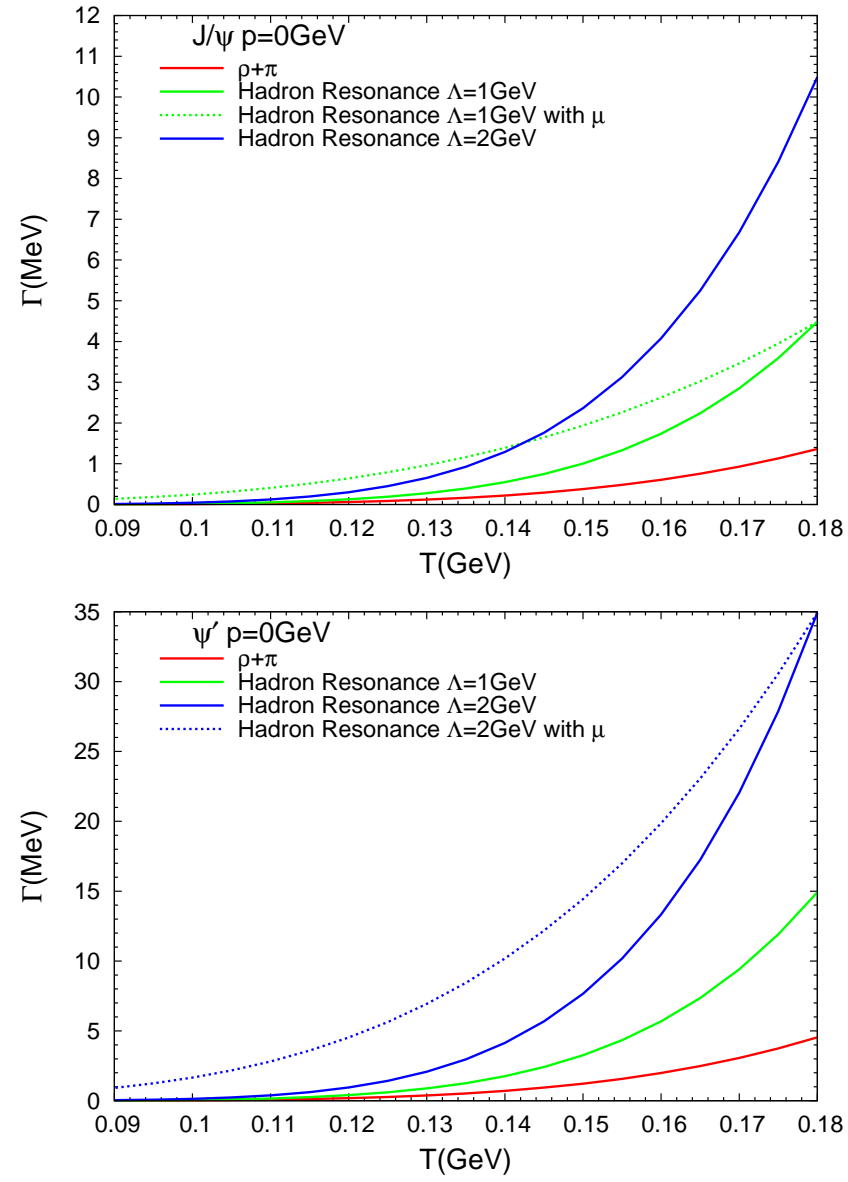

FIG. 1: (Color online) Temperature dependence of hadronic dissociation rates for $J / \psi$ (upper panel) and $\psi^{\prime}$ (lower panel) at rest in a thermal bath. Previous results for a $\pi \rho$ gas with $\Lambda=1 \mathrm{GeV}$ (solid red lines) are compared to our updated results for a meson resonance gas using $\Lambda=1 \mathrm{GeV}$ and $2 \mathrm{GeV}$ (solid green and blue lines, respectively). The blue-dotted lines additionally account for finite meson chemical potential that build up for temperatures below the chemical freezeout in URHICs [24].

with the hadronic formfactor cutoff values, this is too small to affect the $J / \psi$ abundance during the $5-10 \mathrm{fm} / \mathrm{c}$ lifetime of the hadronic phase in URHICs. For the $\psi^{\prime}$, geometric scaling by the vacuum radius has been assumed, increasing its rates by a factor $\left(r_{\psi^{\prime}} / r_{J \psi}\right)^{2}$, which is approximately compatible with constituent-quark model calculations 23].

A hadron resonance gas (HRG), however, contains many more species than $\pi$ and $\rho$. To estimate their impact on the charmonium dissociation rates, we simply adopt the existing $\rho$ - and $\pi$-induced matrix elements and shift their kinematics according to the pertinent 2particle threshold, i.e.,

$$
\Gamma_{X+J / \psi}^{\text {diss }}(T)=\int \frac{d^{3} k}{(2 \pi)^{3}} f^{X}\left(E_{X}(k) ; T\right) \sigma_{X+J / \psi}^{\text {in }}\left(s, s_{\text {thr }}^{X}\right) v_{\text {rel }}
$$




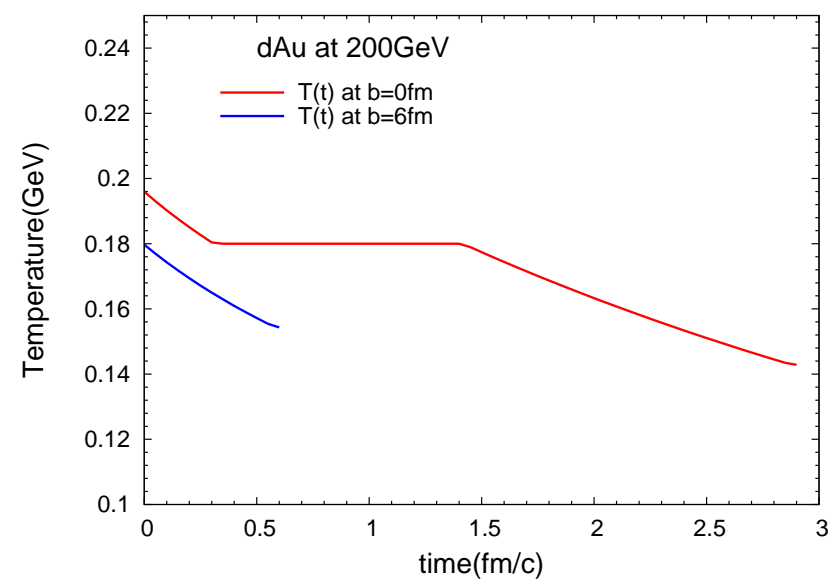

FIG. 2: (Color online) Temperature evolution in $0.2 \mathrm{TeV}$ d$\mathrm{Au}$ collisions assuming a thermal fireball model. The blue (red) curve is for an impact parameter of $b=0(6) \mathrm{fm}$.

with $s=\left(p_{J / \psi}+k\right)^{2}$ and $s_{\mathrm{thr}}^{X}=\left(m_{J / \psi}+m_{X}\right)^{2}$ for exothermic and $s_{\mathrm{thr}}^{X}=\left(2 m_{D}\right)^{2}$ for endothermic channels $\left(s_{\mathrm{thr}}^{X}=\left(m_{D}+m_{D_{s}}\right)^{2}\right.$ if $X$ contains a strange quark $)$. We include a total of 52 non-strange and single-strange meson species, up to a mass of $m_{X}=2 \mathrm{GeV}$. As before, we apply geometric scaling to obtain the reaction rates for the $\chi_{c}$ and $\psi^{\prime}$. Our results for a meson gas in chemical equilibrium are summarized by the solid lines in Fig. 1. At the highest temperature $(T=180 \mathrm{MeV})$, the additional resonances enhance the $J / \psi$ dissociation rate by a factor of $\sim 2.5$, and another factor of $\sim 2.5$ when increasing the hadronic formfactor cutoff from $\Lambda=1 \mathrm{GeV}$ to $2 \mathrm{GeV}$, reaching a maximal rate of $10.5 \mathrm{MeV}$. For the $\psi^{\prime}$, geometric scaling leads a maximum rate of up to $35 \mathrm{MeV}$, translating into a lifetime of $\sim 6 \mathrm{fm} / c$ which is now comparable to the duration of the hadronic phase in URHICs. This becomes even more significant if chemical freezeout is accounted for, which implies the build-up of meson chemical potentials leading to a slower decrease of the meson densities as temperature decreases, cf. dotted lines in Fig. 1)

\section{B. Charmonium Production in $0.2 \mathrm{TeV} \mathrm{d}-\mathrm{Au}$ Collisions}

We proceed by implementing our updated hadronic reaction rates into the thermal-rate equation framework developed in Ref. 25]. Another new aspect relative to our previous work [26] is that we allow for final-state effects in small collision systems, specifically for d-Au at RHIC, where a rather large $\psi^{\prime}$ suppression has been observed (while $J / \psi$ 's are much less suppressed).

Toward this end, we construct a thermal fireball using the same methods as before for AA collisions. We determine the total entropy of the fireball by matching the observed final-state hadron abundances. Based on

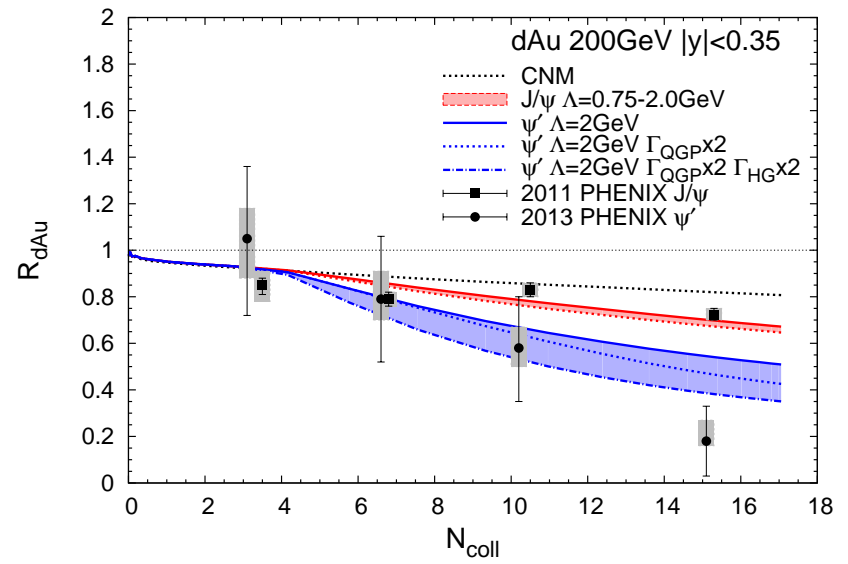

FIG. 3: (Color online) Centrality dependence of the nuclear modification factor for charmonia in $0.2 \mathrm{TeV} \mathrm{d}-\mathrm{Au}$ collisions at RHIC; CNM effects for all charmonia are shown by the black dotted line (consisent with Ref. [16] based on the EPS09 shadowing [29] parametrization); the blue band illustrates uncertainties in both QGP and hadronic dissociation rates of the $\psi^{\prime}$, while the red band illustrates the uncertainties due to the hadronic dissociation of the $J / \psi$ (solid (dotted) line: $\Lambda=0.75(2) \mathrm{GeV})$. The PHENIX data are from Refs. [14, 30].

a Glauber model for the centrality dependent nuclear overlap function, we initialize the fireball with a transverse radius of $R_{0} \simeq 2.5 \mathrm{fm}$. The expansion is then modeled with the same acceleration as in $0.2 \mathrm{TeV} \mathrm{Au}-\mathrm{Au}$ before [26]. For simplicity, we stick with our first-order equation of state, transitioning from a quasi-particle QGP into a HRG through a mixed phase at $T=180 \mathrm{MeV}$ (we do not expect large changes when utilizing a modern cross-over EoS, which has been checked for dilepton [27] and bottomonium observables [28]). Kinetic freezeout is also constructed as before, with a freezeout temperature mildly decreasing with centrality, from $T_{\mathrm{fo}} \simeq 155 \mathrm{MeV}$ in peripheral to $\sim 142 \mathrm{MeV}$ in central collisions, resulting in fireball lifetimes of $0.5-3 \mathrm{fm} / c$, cf. Fig. 2, For the most central collisions, a short QGP phase with initial temperature $T_{0}=190 \mathrm{MeV}$ is followed by a $1 \mathrm{fm} / c$ mixed phase and a $1.5 \mathrm{fm} / c$ hadronic phase.

The initial CNM effects are assumed to be identical for all charmonia, essentially given by a shadowing suppression [16] which we mimic with an "effective" nuclear absorption cross section of $\sigma_{\mathrm{abs}}=2.4 \mathrm{mb}$. Our results for the centrality dependence of the nuclear modification factor,

$$
R_{\mathrm{AA}}^{\Psi}\left(N_{\text {part }}\right)=\frac{N_{\Psi}\left(N_{\text {part }}\right)}{N_{\Psi}^{p p} N_{\text {coll }}\left(N_{\text {part }}\right)}
$$

are summarized for both $\Psi=J / \psi, \psi^{\prime}$ in Fig. 3, in comparison to PHENIX data 14] $\left(N_{\text {coll }}\right.$ : number of primordial $N N$ collisions). For the $J / \psi$, there is a moderate suppression beyond CNM effects; the additional finalstate effects are due to a small QGP suppression on the direct $J / \psi$ 's, as well as a suppression of the feeddown 
from $\chi_{c}$ 's and $\psi^{\prime}$, with little room for additional hadronic suppression (thus, in principle, preferring a small value for the hadronic cutoff parameter). On the other hand, for the $\psi^{\prime}$, our baseline QGP+HRG suppression is not enough to account for the marked suppression beyond CNM effects. Here, a large formfactor cutoff of $\Lambda=2 \mathrm{GeV}$ is preferred to augment the hadronic suppression. An additional increase of the QGP suppression rate of the $\psi^{\prime}$ by a factor of 2 could also be helpful (such an increase may arise, e.g., from nonperturbative heavy-quark interactions with light partons). Further increasing the hadronic rate by a factor of $2^{1}$ has a similar effect, reducing the discrepancy with the most central PHENIX datum.

We finally note that a hadronic $\psi^{\prime}$ dissociation rate well beyond the one from the $\pi \rho$ gas (by a factor of 5 or more) was previously deduced within our setup 13] to be able to account for the $\psi^{\prime}$ suppression observed in $\mathrm{S}-\mathrm{U}$ and $\mathrm{Pb}-\mathrm{Pb}$ collisions at the SPS [10]. Our newly calculated rates in the present $\mathrm{ms}$. are in line with this notion.

\section{SEQUENTIAL REGENERATION OF CHARMONIA}

In this section, we investigate the consequences of the updated hadronic reaction rates within our previously constructed thermal fireball expansion (Sec. III A), followed by a more generic evaluation of the associated uncertainties specifically in the context of the $\psi^{\prime} / J / \psi R_{\mathrm{AA}}$ double ratio (Sec. IIIB). We focus on $2.76 \mathrm{TeV} \mathrm{Pb}-\mathrm{Pb}$ collisions at the LHC. Our earlier predictions for inclusive $J / \psi$ production in these reactions [5] resulted in a fair agreement with the centrality, transverse-momentum and rapidity dependencies observed by the ALICE and CMS collaborations, and thus serves as our framework to evaluate $\psi^{\prime}$ observables. Since the pertinent CMS data are for "prompt" $J / \psi$ and $\psi^{\prime}$ production, we do not include contributions from $B$ feeddown.

\section{A. Fireball Model}

For our fireball results we focus on the so-called "strong-binding scenario", where the in-medium charmonium properties are taken with guidance from a $T$-matrix approach [31] with the internal energy from lattice-QCD as underlying potential. This assumption gives a better agreement than using the free energy both with correlators from lattice-QCD and the overall charmonium phenomenology at SPS and RHIC [26]. For definiteness, we

1 This could be due to inelastic reactions with baryons and antibaryons or direct $\psi^{6} \rightarrow D \bar{D}$ decays with an in-medium reduced $D$ meson mass [25], neither of which we have calculated here.

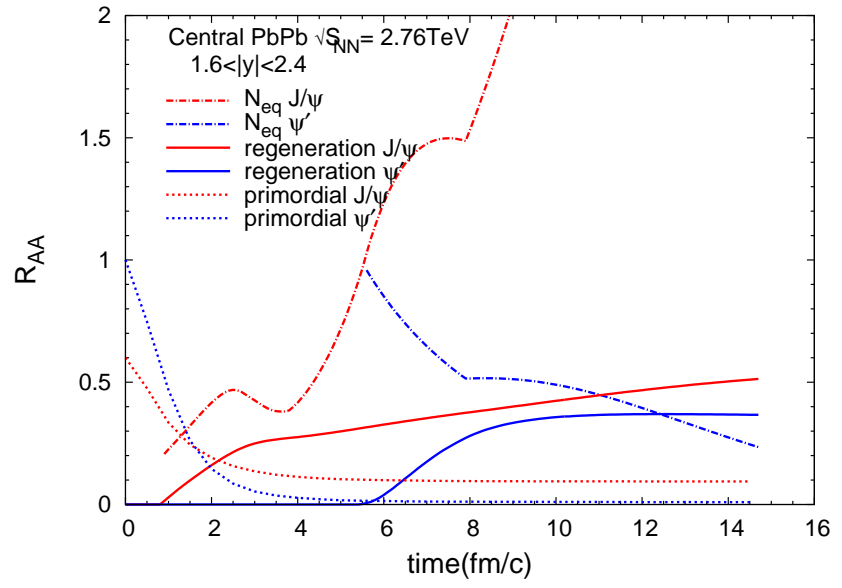

FIG. 4: (Color online) Time dependence of $J / \psi$ and $\psi^{\prime}$ nuclear modification factors in $\operatorname{central}\left(N_{\text {part }}=324\right) \mathrm{Pb}-\mathrm{Pb}$ collisions at $\sqrt{s}=2.76 \mathrm{TeV}$ (the denominator for the $J / \psi R_{\mathrm{AA}}$ includes $40 \%$ feeddown from excited states while the numerator does not). The red (blue) curves are for direct $J / \psi\left(\psi^{\prime}\right)$, with the solid (dotted) line styles representing the regeneration (primordial) contributions; the dash-dotted curves indicate the pertinent equilibrium limits (including a thermal relaxation time correction [13]), starting from the time when the fireball has cooled down to the dissociation temperature below which regeneration commences.

employ the hadronic rates with $\Lambda=0.75(2) \mathrm{GeV}$ for the $J / \psi\left(\psi^{\prime}\right)$, and a factor of 2 increased QGP rate of the $\psi^{\prime}$.

Let us first inspect the time evolution of direct $J / \psi$ and $\psi^{\prime}$ mesons in $0-20 \%$ central $\mathrm{Pb}-\mathrm{Pb}$ (without shadowing), as following from the solution of the kinetic rate equation,

$$
\frac{N_{\Psi}}{d \tau}=-\Gamma_{\Psi}\left[N_{\Psi}(\tau)-N_{\psi}^{\mathrm{eq}}(T(\tau))\right],
$$

see Fig. 4. Compared to our previous results (cf. lower panel in Fig. 2 of Ref. [5]), the $J / \psi$ now picks up a regeneration contribution in the hadronic phase, by about 0.15 units in $R_{\mathrm{AA}}$, which, despite a small rate, is due to the large equilibrium limit. Most of the production, however, occurs prior to the onset of the mixed phase at $\tau \simeq 5.5 \mathrm{fm} / c$. On the contrary, $\psi^{\prime}$ production only starts to set in at that point, leveling off at around $\tau \simeq 9$ $10 \mathrm{fm} / c$, when the temperature of the fireball has dropped to about $150-160 \mathrm{MeV}$. The main qualitative and robust feature here is that the lower dissociation temperature of the $\psi^{\prime}$, relative to the $J / \psi$, implies a later production through regeneration in the time evolution of the fireball in URHICs.

Since the equilibrium limit for the charmonia $R_{\mathrm{AA}}$ is a key input to the rate equation, let us recall how we calculate it. As an example, we consider the $\psi^{\prime}$ in central $\mathrm{Pb}-\mathrm{Pb}$ collisions $(b \simeq 4.1 \mathrm{fm})$ as displayed in Fig. 4 With a $N_{\text {coll }}$-weighted collision number of $\bar{N}_{\text {coll }}=1312$, an inelastic $p p$ cross section of $\sigma_{\text {inel }}=65 \mathrm{mb}$ and $\left.\frac{d \sigma_{c \bar{c}}^{p p}}{d y}\right|_{y<1.6}=0.75 \mathrm{mb}$ (no shadowing) extrapolated to slightly forward rapidity as $\left.\frac{d \sigma_{c c}^{p p}}{d y}\right|_{1.6<y<2.4} \simeq 0.65 \mathrm{mb}$, we 
obtain $N_{c \bar{c}} \approx 24$ over the rapidity range of $\Delta y \approx 1.8$ as covered by a thermal fireball. From this number, we compute a charm fugacity factor $\gamma_{c}$ by matching it to the total charm density using the charm conservation relation [11]

$$
N_{c \bar{c}}=\frac{1}{2} \gamma_{c} n_{\mathrm{open}} V_{\mathrm{FB}} .
$$

For simplicity, we here neglect the small contributions from charmonia (they are included in our full calculations) and work in the grand canonical limit. For definiteness, we take the fireball evolution time at $\tau=8 \mathrm{fm} / c$ where the medium has just completed hadronization at $T_{c}=180 \mathrm{MeV}$ (beginning of the hadronic phase) with a fireball volume of $V_{\mathrm{FB}} \approx 3000 \mathrm{fm}^{3}$. The total thermal open-charm density, $n_{\text {open }}$, is obtained by summing over $10 D$ and $D_{s}$ mesons (and their anti-particles) up to masses of $m_{D}=2.6 \mathrm{GeV}$ and 19 charmed (anti-) baryons up to $m_{B}=3 \mathrm{GeV}$, resulting in $n_{\text {open }} \approx 0.00084 \mathrm{fm}^{-3}$. From Eq. (4) one then obtains $\gamma_{c} \approx 19$. With $n_{\psi^{\prime}}(T=180 \mathrm{MeV}) \approx 9.8 \cdot 10^{-9} \mathrm{fm}^{-3}$, one finds $N_{\psi^{\prime}}^{e q}=\gamma_{c}^{2} n_{\psi^{\prime}} V_{\mathrm{FB}} \mathcal{R}(\tau) \approx 0.009$ (where the correction factor, $\mathcal{R}(\tau) \simeq 0.83$, accounts for incomplete charmquark thermalization 13]|). To convert that into an $R_{\mathrm{AA}}$, one needs to compute the $\bar{N}_{\text {coll-scaled }} \psi^{\prime}$ number, $N_{\psi^{\prime}}^{p p} \bar{N}_{\text {coll }}$, figuring into the denominator. Using a $J / \psi p p$ cross section of $\left.\frac{d \sigma_{J / \psi}^{p p}}{d y}\right|_{1.6<y<2.4} \simeq 3.5 \mu b$ (extrapolated from $\left.\left.\frac{d \sigma_{J / \psi}}{d y}\right|_{y<1.6}=4 \mu b\right)$, yields $N_{\psi^{\prime}} \bar{N}_{\text {coll }}=$ $14 \% N_{J / \psi}^{p p} \bar{N}_{\text {coll }} \simeq 0.018$ within the 1.8 rapidity units covered by the fireball. This finally results in an estimate of $R_{\mathrm{AA}}^{e q}\left(\psi^{\prime}\right)=\frac{N_{\psi^{\prime}}^{e q}}{N_{\psi^{\prime}}^{p p} N_{\text {coll }}} \approx 0.5$, quite close to the more precisely calculated value of 0.52 shown in Fig 4 The $J / \psi$ equilibrium value for $R_{\mathrm{AA}}$ at $T=180 \mathrm{MeV}$ readily follows from $N_{\psi}^{e q} / N_{\psi^{\prime}}^{e q} \approx 20.5$ as $R_{\mathrm{AA}}^{J / \psi} \approx 14 \% \cdot 20.5 \cdot R_{\mathrm{AA}}^{\psi^{\prime}} \approx 1.5$.

The sequential regeneration of $J / \psi$ and $\psi^{\prime}$ has rather dramatic consequences on their transverse-momentum $\left(p_{t}\right)$ spectra. Following our previous work [9], we approximate the $p_{t}$ spectra of the regeneration components with the standard blast-wave expression,

$\frac{d N_{\Psi}^{\mathrm{reg}}}{p_{t} d p_{t}}=N_{0}(b) m_{t} \int_{0}^{R} r d r K_{1}\left(\frac{m_{t} \cosh \rho(r)}{T}\right) I_{0}\left(\frac{p_{t} \sinh \rho(r)}{T}\right)$

implying thermalized charm-quark distributions. The $m_{t}=\sqrt{p_{t}^{2}+m^{2}}$ is the transverse mass. As our default, we evaluate this expression when most of the pertinent charmonium yield has built up, i.e., at $T_{c}$ for the $J / \psi$ $\left(\tau=5.5 \mathrm{fm} / c\right.$ in Fig. (4) and at $T=155 \mathrm{MeV}$ for the $\psi^{\prime}$ ( $\tau=9.7 \mathrm{fm} / c$ in Fig. (4). The resulting nuclear modification factors, $R_{\mathrm{AA}}\left(p_{t}\right)$, for both regeneration and surviving primordial components are displayed in Fig. 5 (we have assumed the same initial spectra for $J / \psi$ and $\psi^{\prime}$ from $p p$ collisions, figuring into the denominator of $\left.R_{\mathrm{AA}}\right)$. Clearly, there is a significant uncertainty associated with this procedure which we address in Sec. IIIB below (we recall, however, that our pertinent predictions

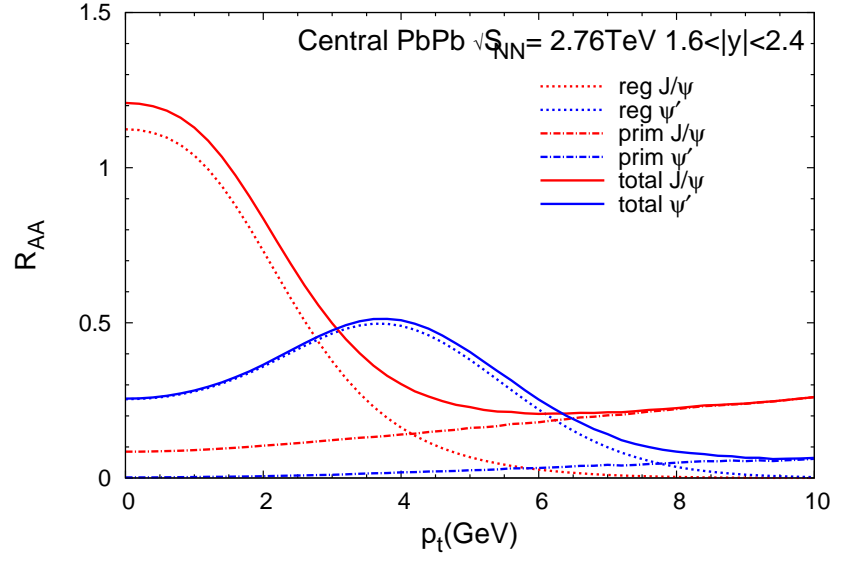

FIG. 5: (Color online) Nuclear modification factor for $J / \psi$ (red lines) and $\psi^{\prime}$ mesons (blue lines) as a function of $p_{t}$ for 0 $20 \% \mathrm{~Pb}-\mathrm{Pb}(2.76 \mathrm{TeV})$ collisions. The total $R_{\mathrm{AA}}$ for each meson (solid lines) is decomposed in a regeneration component (dotted lines, evaluated with a blast-wave ansatz in the fireball evolution at sequential freezeout times) and a suppressed primordial component (dash-dotted lines, as obtained from a Boltzmann transport equation without gain term), cf. Ref. 9] for further details.

for the $J / \psi$ gave fair agreement with the observed $p_{t}$ spectra at LHC). Three main qualitative features can be gleaned from comparing the $R_{\mathrm{AA}}$ 's for $J / \psi$ and $\psi^{\prime}$ in Fig. 55 (a) At low $p_{t} \lesssim 3 \mathrm{GeV}$, the regeneration yield of the $J / \psi$ dominates over the one from $\psi^{\prime}$, as a consequence of the approach toward equilibrium which favors the smaller $J / \psi$ mass; (b) At intermediate $p_{t} \simeq 3-6 \mathrm{GeV}$, the significantly harder blast wave for the $\psi^{\prime}$ generates a shift of the "flow bump" which exceeds the regeneration contribution in the $J / \psi R_{\mathrm{AA}}$; (c) at still higher momenta, $p_{t} \gtrsim 6-8 \mathrm{GeV}$, regeneration gives way to (suppressed) primordial production, where the $J / \psi R_{\mathrm{AA}}$ exceeds again the one from the more weakly bound $\psi^{\prime}$. Items (b) and (c) are in qualitative agreement with the trends observed in the pertinent $R_{\mathrm{AA}}^{\psi^{\prime}} / R_{\mathrm{AA}}^{J / \psi}$ double ratio observed by CMS [18]. In the next section we explore some of the uncertainties in our calculations.

\section{B. Schematic Model}

To better quantify variations in the interplay of the different production components of both $J / \psi$ and $\psi^{\prime}$, let us first formulate a baseline scenario motivated by existing experimental data for the $J / \psi$ and the thermal fireball calculations in the previous sections. For simplicity, we assume the primordial parts to be constant in $R_{\mathrm{AA}}\left(p_{t}\right)$. This reflects our currently limited knowledge about the $p_{t}$ dependence of the dissociation rates (see, e.g., Ref. [9]), formation time effects, etc. For $0-20 \% \mathrm{~Pb}-\mathrm{Pb}$ collision we take $0.15-0.25$ for the $J / \psi$ (compatible with high- $p_{t}$ CMS data for prompt $J / \psi[32]$ ) and $0-0.075$ for the $\psi^{\prime}$, to re- 


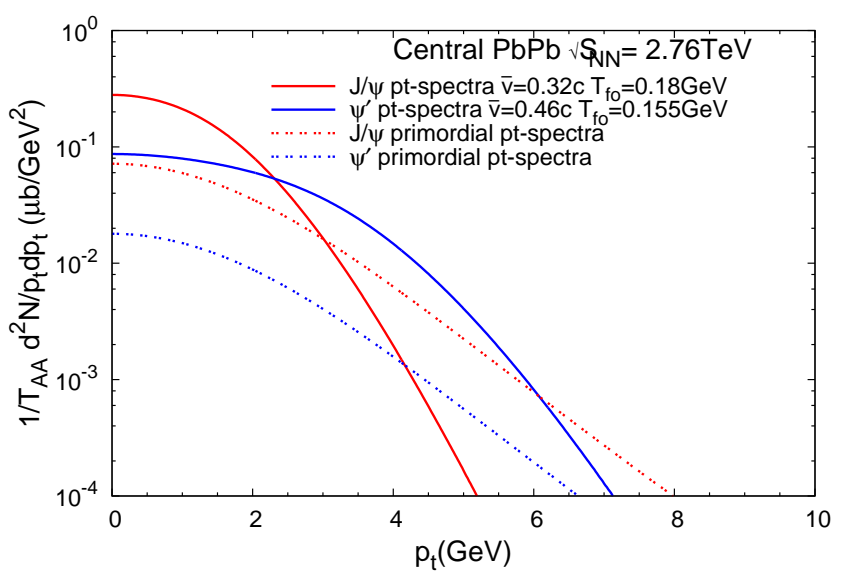

FIG. 6: (Color online) Transverse-momentum spectra of $J / \psi$ (red lines) and $\psi^{\prime}$ mesons (blue lines) for both suppressed primordial (dashed lines, assuming $R_{\mathrm{AA}}^{J / \psi}=0.2$ and $R_{\mathrm{AA}}^{\psi^{\prime}}=0.05$ ) and regeneration components (solid lines); the total yields (not the shapes) are normalized to their $N_{\text {coll-scaled number }}$ in $p p$ collisions.

flect its stronger absorption as a loosely bound state. For the regeneration components, we choose total yields such that the total momentum-integrated $R_{\mathrm{AA}}$ amounts to 0.55-0.65 for the $J / \psi$ (compatible with ALICE data 33]) and 0.4 for the $\psi^{\prime}$ (as suggested by our fireball results). For the sequential freezeout, which determines the temperature and flow strength in the blast-wave spectra of the regeneration components, we employ the correlation given by the expanding fireball, i.e., $\tau \simeq 5.5(9.7) \mathrm{fm} / c$ for the $J / \psi\left(\psi^{\prime}\right)$ corresponding to $T_{\mathrm{fo}}=180(155) \mathrm{MeV}$ and $\bar{v}=0.32(0.46) c$. Recall that the effective slope parameter for the blast-wave spectra is approximately given by $T_{\text {eff }} \approx T+m_{\Psi} \bar{v}^{2}$, which is mostly driven by the flow term due to the large mass of the charmonia. In Fig. 6 we display the pertinent $p_{t}$ spectra for central $\mathrm{Pb}-\mathrm{Pb}(2.76 \mathrm{TeV})$,

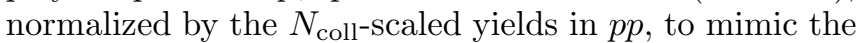
relative magnitude of $\psi^{\prime}$ and $J / \psi$ contributions in their respective $R_{\mathrm{AA}}$ 's. The plot highlights again the main effect proposed in this paper: due to the stronger flow for the regenerated $\psi^{\prime}$, its $R_{\mathrm{AA}}$ can rise above the one for the $J / \psi$ in a limited $p_{t}$ window around $3-6 \mathrm{GeV}$, before primordial production takes over again.

For semi-central $\mathrm{Pb}-\mathrm{Pb}(20-40 \%)$ we construct the baseline following the same reasoning as for central collisions. We increase the primordial $R_{\mathrm{AA}}$ 's for $J / \psi$ to 0.35 0.45 [32] and to 0.1-0.2 for $\psi^{\prime}$, and evaluate the blastwave $p_{t}$ spectra at the same freezeout temperatures as for central collisions (but with flow velocities given by the fireball expansion for $20-40 \%$ centrality).

The resulting double ratios covering the abovespecified ranges in the primordial components are shown in the upper panel of Fig. 7 and compared to CMS data [18] (we did not include the ALICE data [19] as they contain feeddown contributions from $B$-meson decays, which could become rather significant at high $p_{t}$,
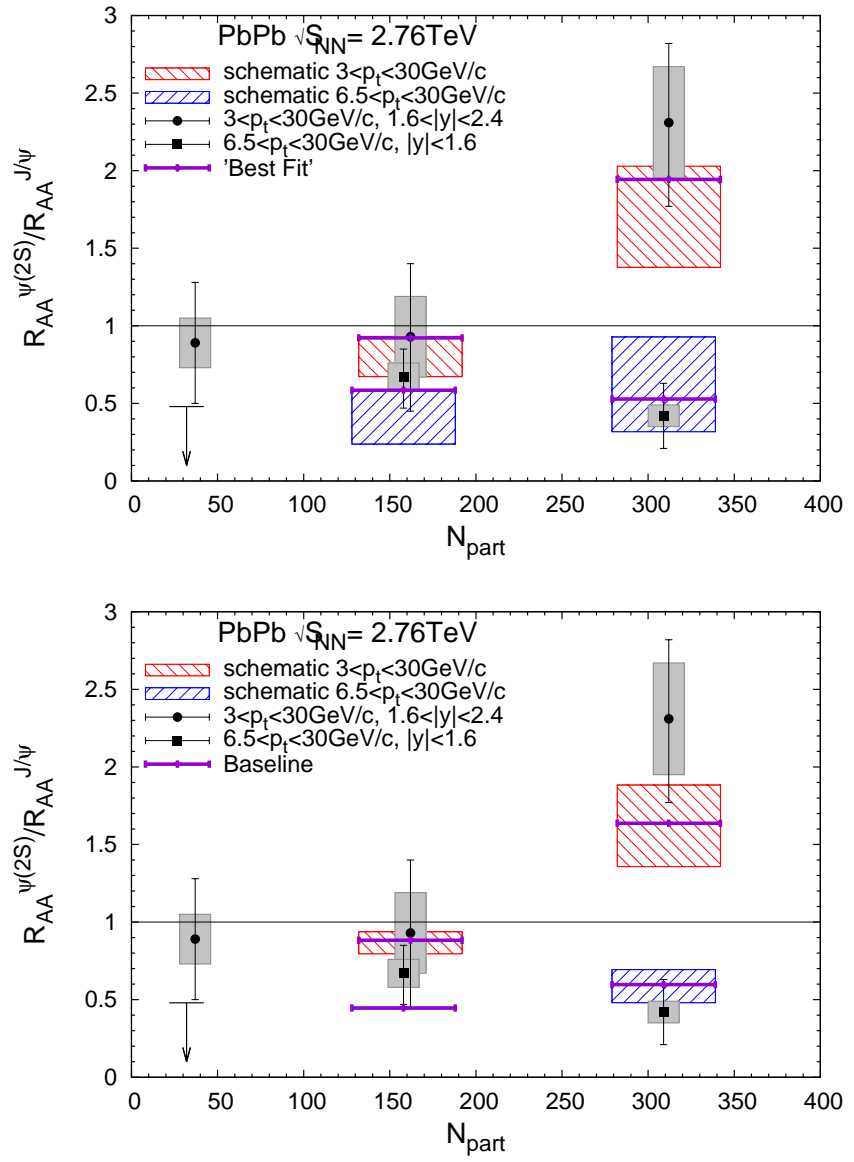

FIG. 7: (Color online) Ratio of the $R_{\mathrm{AA}}$ for $\psi^{\prime}$ to the one for $J / \psi$ (the so-called "double ratio") as a function of centrality in $2.76 \mathrm{TeV} \mathrm{Pb}-\mathrm{Pb}$ collisions. The red and blue boxes are our schematic-model results for the double ratio in the momentum range $p_{t}=3-30 \mathrm{GeV}$ and $p_{t}=6.5-30 \mathrm{GeV}$, respectively, and are compared to CMS data [18]. In the upper (lower) panel, the boxes indicate theoretical uncertainties in the modeling of the primordial (regeneration) component of the charmonium yields and spectra. The vertical purple lines represent our "best fit" in the upper panel, and a "realistic" baseline for the blast-wave variations in the lower panel.

see, e.g., Ref. 20] for a calculation including those). The basic trends of the data can be reproduced within our approach. For definiteness, we quote (approximate) "best fit" values of 0.15 and $\sim 0(0.35$ and 0.2$)$ for the primordial $J / \psi$ and $\psi$ ' values, respectively, in (semi-) central $\mathrm{Pb}-\mathrm{Pb}$ collisions, resulting in the purple horizontal bars in the upper panel of Fig. 7

Finally, we illustrate the sensitivity of the double ratios to the blast-wave parameters, by varying the freezeout temperatures over the ranges $T_{\mathrm{fo}}^{J / \psi}=180-200 \mathrm{MeV}$ and $T_{\mathrm{fo}}^{\psi^{\prime}}=150-165 \mathrm{MeV}$, along with the pertinent flow velocities from the fireball model, and fixing the primordial components at $R_{\mathrm{AA}}^{J / \psi}=0.2(0.35)$ and $R_{\mathrm{AA}}^{\psi^{\prime}}=0.05(0.15)$ for (semi-) central $\mathrm{Pb}-\mathrm{Pb}$. The larger regeneration components and larger flow velocities in central collisions render 
the double ratios more sensitive to the details of the sequential regeneration mechanism.

\section{CONCLUSIONS}

In the present work, we have investigated the production systematics of $\psi^{\prime}$ mesons in URHICs. We first revisited the problem of hadronic $\psi^{\prime}$ dissociation and found that a more complete inclusion of hadronic states in a resonance gas suggests a marked increase of its inelastic reaction rates. When implementing these rates into an expanding fireball for $\mathrm{d}-\mathrm{Au}$ collisions at RHIC, we found a much improved description of the rather strong suppression of $\psi^{\prime}$ mesons observed in these reactions. This is similar in spirit to, and thus supports, the recently suggested comover suppression effects [16] in $\mathrm{dA}$ and $p \mathrm{~A}$ reactions at RHIC and $\mathrm{LHC}$. We then evaluated $\psi^{\prime}$ transport in $\mathrm{Pb}-\mathrm{Pb}$ collisions at the LHC using our existing rate equation approach which previously provided fair agreement with $J / \psi$ data. The key features in our approach are the lower dissociation temperature of the $\psi^{\prime}$ relative to the $J / \psi$ and its sizable hadronic reaction rates. This implies a sequential freezeout of these two mesons, with most of the $\psi^{\prime}$ regeneration occurring later in the fireball evolution. The larger collective medium flow then leads to an enhancement of the $\psi^{\prime}$ regeneration yield in a $p_{t}$ region around $3-6 \mathrm{GeV}$, transitioning to (suppressed) primordial production at higher $p_{t}$. While quantitative predictions of this mechanism are beyond current theoretical control, we have shown that variations in the ingredients to the sequential regeneration scenario produce trends in the $\psi^{\prime}$-over- $J / \psi R_{\mathrm{AA}}$ double ratio which agree with recent CMS data. We therefore believe that the qualitative features of this mechanism are robust and provide a candidate to contribute to the understanding of these data. In fact, if corroborated, sequential regeneration may serve as a tool to extract in-medium properties of the $\psi^{\prime}$ from URHIC data.

\section{Acknowledgments}

We are indebted to Xingbo Zhao for providing us with his codes and valuable discussions. This work is supported by the US National Science Foundation under grant no. PHY-1306359.
[1] T. Matsui and H. Satz, Phys. Lett. B178, 416 (1986).

[2] R. Rapp, D. Blaschke, and P. Crochet, Prog. Part. Nucl. Phys. 65, 209 (2010).

[3] L. Kluberg and H. Satz, Landolt-Bornstein 23, 372 (2010), [arXiv:0901.3831].

[4] P. Braun-Munzinger and J. Stachel, Landolt-Bornstein 23, 424 (2010), [arXiv:0901.2500].

[5] X. Zhao and R. Rapp, Nucl. Phys. A859, 114 (2011).

[6] K. Zhou, N. Xu, Z. Xu, and P. Zhuang, Phys. Rev. C89, 054911 (2014).

[7] T. Song, K. C. Han, and C. M. Ko, Phys. Rev. C84, 034907 (2011).

[8] A. Andronic, P. Braun-Munzinger, K. Redlich, and J. Stachel, J. Phys. G37, 094014 (2010).

[9] X. Zhao and R. Rapp, Phys. Lett. B664, 253 (2008).

[10] M. Abreu et al. (NA50), Nucl. Phys. A638, 261 (1998).

[11] P. Braun-Munzinger and J. Stachel, Phys. Lett. B490, 196 (2000).

[12] H. Sorge, E. V. Shuryak, and I. Zahed, Phys. Rev. Lett. 79, 2775 (1997).

[13] L. Grandchamp and R. Rapp, Nucl. Phys. A709, 415 (2002).

[14] A. Adare et al. (PHENIX), Phys. Rev. Lett. 111, 202301 (2013).

[15] B. B. Abelev et al. (ALICE), JHEP 1412, 073 (2014).

[16] E. G. Ferreiro, Phys. Lett. B749, 98 (2015).

[17] Y. Liu, C. M. Ko, and T. Song, Phys. Lett. B728, 437 (2014).
[18] V. Khachatryan et al. (CMS), Phys. Rev. Lett. 113, 262301 (2014).

[19] R. Arnaldi (ALICE), Nucl. Phys. A904-905, 595c (2013).

[20] B. Chen, Y. Liu, K. Zhou, and P. Zhuang, Phys. Lett. B726, 725 (2013).

[21] Z.-w. Lin and C. Ko, Phys. Rev. C62, 034903 (2000).

[22] K. L. Haglin and C. Gale, Phys. Rev. C63, 065201 (2001).

[23] T. Barnes, E. Swanson, C. Wong, and X. Xu, Phys. Rev. C68, 014903 (2003).

[24] R. Rapp, Phys. Rev. C66, 017901 (2002).

[25] L. Grandchamp, R. Rapp, and G. E. Brown, Phys. Rev. Lett. 92, 212301 (2004).

[26] X. Zhao and R. Rapp, Phys. Rev. C82, 064905 (2010).

[27] R. Rapp, Adv. High Energy Phys. 2013, 148253 (2013).

[28] A. Emerick, X. Zhao, and R. Rapp, Eur. Phys. J. A48, 72 (2012).

[29] K. Eskola, H. Paukkunen, and C. Salgado, JHEP 0904, 065 (2009).

[30] A. Adare et al. (PHENIX), Phys. Rev. Lett. 107, 142301 (2011).

[31] F. Riek and R. Rapp, Phys. Rev. C82, 035201 (2010).

[32] S. Chatrchyan et al. (CMS), JHEP 1205, 063 (2012).

[33] B. B. Abelev et al. (ALICE), Phys. Lett. B734, 314 (2014). 